\title{
Edmond Jabès y Eduardo Chillida: La Mémoire et la Main
}

\author{
Francisco Javier DECO PRADOS \\ Universidad de Cádiz \\ Departamento de Filología Francesa e Inglesa \\ fjavier.deco@uca.es
}

Recibido: $31 / 05 / 2013$

Aceptado: 19/02/2014

\section{Resumen}

Se estudian aquí varios aspectos de la obra La Mémoire et la Main, fruto de la colaboración entre Edmond Jabès y Eduardo Chillida. Analizo el proceso de sus ediciones desde la publicación del primer poema en Orange Export Ltd. hasta la aparición de una segunda edición en 1987 en la editorial Fata Morgana, con la reproducción simplificada de los aguafuertes. Basándome sobre todo en la edición de 1986, atiendo al soporte (papel, encuadernación) y a la mise en page (tipografía, disposición, etc.) y me detengo especialmente en el estudio de las ilustraciones. Intento poner en relación el ámbito de los aguafuertes y el contenido del texto y realizar una reflexión sobre la funcionalidad estética de los distintos elementos del libro en su interrelación.

Palabras clave: Edmond Jabès, Eduardo Chillida, poesía francesa del siglo XX, libros ilustrados, estética del libro.

\section{Edmond Jabès et Eduardo Chillida: La Mémoire et la Main}

\section{Résumé}

Il s'agit ici d'étudier plusieurs aspects de l'ouvrage La Mémoire et la Main, fruit de la collaboration entre Edmond Jabès et Eduardo Chillida. J'en analyse les différentes éditions depuis la publication du premier poème par Orange Export Ltd. jusqu'à la réédition de 1987 chez Fata Morgana, contenant une reproduction simplifiée des eaux-fortes de Chillida. En me fondant principalement sur l'édition de Lelong (1986), j'étudie le support -papier, reliure- et la mise en page, et je m'attache plus particulièrement aux illustrations. Je tente de mettre en relation l'univers des eaux-fortes et le contenu du texte et d'en tirer une réflexion sur la fonctionnalité esthétique des différents éléments du livre dans leur interaction.

Mots clés: Edmond Jabès, Eduardo Chillida, poésie française du $\mathrm{XX}^{\mathrm{e}}$ siècle, livres illustrés, esthétique du livre.

\section{Edmond Jabès and Eduardo Chillida: La Mémoire et la Main}

\begin{abstract}
Several aspects of the work La Mémoire et la Main, fruit of the collaboration between Edmond Jabès and Eduardo Chillida are the object of our study. I aim to analyze the process of its editions, from the publication of the first poem in Orange Export Ltd. to the appearance of a second edition in 1987
\end{abstract}


published by Fata Morgana with a simplified reproduction of the etchings. Basing my findings primarily on the 1986 edition, I examine the format (paper, binding) and the mise en page (typography, page layout, etc.), with a particular analytical emphasis on the illustrations. I attempt to locate the connections between the domain of the etchings and the content of the text so as to consider the aesthetic functionality of the diverse elements of the book and their connections with one another.

Key words: Edmond Jabès, Eduardo Chillida, twentieth century French poetry, illustrated books, book aesthetics.

\section{Referencia normalizada}

Deco Prados, F. J. (2014). "Edmond Jabès y Eduardo Chillida: La Mémoire et la Main". Thélème. Revista Complutense de Estudios Franceses, Vol. 29, Núm. 2: 293-308. http://dx.doi.org/10.5209/rev_THEL.2014.v29.n2.42389

Eduardo Chillida realiza en 1956 su primera exposición individual en Francia ${ }^{1}$ en la galería de Aimé Maeght (donde ya había expuesto dos obras en 1950), acompañada del catálogo correspondiente en la serie Derrière le miroir ${ }^{2}$, con texto de Gaston Bachelard. La relación con la galería será muy fecunda durante muchos años. La colaboración entre el escultor y Jabès comienza en 1975, año en que aparece un placard con tres aforismos de éste junto a un grabado de Chillida, editado justamente por Maeght. Se trata de una litografía original de 60 x $96,5 \mathrm{~cm}$ tirada a 500 ejemplares, numerada y firmada por los autores, que formaba parte de una colección en la que el galerista editor buscaba la coparticipación de artistas y escritores $^{3}$.

${ }^{1}$ Chillida (1924-2002) se traslada a París en 1948, donde su carrera artística empieza a cobrar forma. Este mismo año, el Musée d'Art Moderne de la ciudad elige una obra suya para el Salón de mayo. En 1950, tras su boda, el escultor se instala junto a su esposa en la región parisina. Aunque en 1951 vuelve a España definitivamente, seguirá haciendo frecuentes viajes a París. Su relación con Francia continuará siendo estrecha en lo sucesivo, tanto en el plano profesional como personal. Cfr. Museo Chillida Leku, "Biografía".

${ }^{2}$ Publicación que servirá como lugar de encuentro entre poetas, ensayistas y artistas ideada por el creador de la galería en 1946. Los artistas realizaban litografías originales para ilustrar los números de DLM. El mítico taller Mourlot se ocupó de estas impresiones y la no menos célebre imprenta Union de los textos, desde el número 4 al 115 (1959).

${ }^{3}$ Las tres frases bajo el dibujo (todo en blanco y negro) son: "Le trait est désir exaucé d'un point pour un autre point. Le plus court chemin. // Le premier souffle vient du plus lointain passé; le dernier souffle lui doit encore sa tiédeur. // Ce qui ne se laisse pas saisir est éternel". Cfr. Maeght Éditions, "Chillida. Placard Jabès".

Cfr. una descripción de la mayoría de los libros ilustrados del autor en Biblioteca Nacional (2007). Por otra parte, hemos de subrayar que la poesía ha sido siempre un género especialmente apreciado por Chillida, siendo numerosas las colaboraciones realizadas con poetas de expresión francesa (vide infra). 
Es preciso indicar que a partir de ese año de 1975 se intensifica progresivamente la colaboración de Jabès con artistas ${ }^{4}$. Esto no era del todo una novedad en su carrera, ya que en sus primeros libros, de los años 30, la idea de relación con la plástica estaba ya presente ${ }^{5}$. En los años ochenta el número de sus publicaciones ilustradas crece claramente ${ }^{6}$. Es interesante señalar también la relación de Jabès, en este decenio, con la música: en 1980 se estrena Quelques pages du Livre des Questions, de Jean-Yves Bosseur ${ }^{7}$ y en 1987 la obra de Luigi Nono Découvrir la subversion. Hommage à Edmond Jabès ${ }^{8}$.

Es en 1986 cuando las afinidades entre Jabès y Chillida se plasman en un libro ilustrado de gran importancia, La Mémoire et la Main ${ }^{9}$, editado por la galería de la

\footnotetext{
${ }^{4}$ En 1975 aparecen también Ça suit son cours, con Antoni Tàpies, Montpellier, Fata Morgana. En
} 1977 aparece una carpeta gráfica con cinco serigrafias de Eusebio Sempere sobre "La transparence du temps", poema de Elya, traducido por José Miguel Ullán.

${ }_{5}$ Maman, cubierta de Neroni, dibujos de S. J. Dimos, El Cairo, Éditions de La Semaine égyptienne, 1932; Les Pieds en l'air, cubierta et dibujos de Mayo, El Cairo, Alejandría, Éditions de La Semaine égyptienne, s.d. 1934; Arrhes poétiques, dibujos del autor por Jean Moscatelli, El Cairo, Éditions de La Semaine égyptienne, 1935. Tras esta primera fase se abre un largo paréntesis sólo roto en 1951 con Les Mots tracent, aguafuerte de Jacques Villon, cubierta de Max Ernst, París, Librairie Les Pas Perdus, "L'Âge d'or".

${ }^{6}$ Hasta 1991, año de la muerte de Jabès: Récit, con ilustraciones de Jean Degottex, Montpellier, Fata Morgana, 1982. Surcharge, con dos obras de Francesca Chandon, 1983. Dans la double dépendance du dit. Le Livre des Marges II, ilustraciones de Antoni Tàpies, Saint-Clément-de-Rivière, Fata Morgana, 1984 (también con Tàpies aparecerá en 1997 Bâtir au quotidien. Le Livre des Marges III, esquisse, Saint-Clément-de-Rivière, Fata Morgana). Aigle et chouette, con grabados de Jean Capdeville, Saint-Clément-de-Rivière, Fata Morgana, 1986. En 1986-87 el libro con Chillida. En 1989 L'Etranger, con una pintura de Antonio Saura, París, Éditions Marchant Ducel, La Mémoire des mots. Comment je lis Paul Celan, ilustraciones de Gisèle Celan-Lestrange, París, Fourbis, 1990. Jabès/Colomb, texto de Jabès, fotografías de Denise Colomb, París, Éd. Aux dépens d'un exemplaire, 1990. Bâtir à chaux et à sable (páginas de Un étranger avec, sous le bras, un livre de petit format), con grabados de Olivier Debré, París, Le Nouveau cercle parisien du livre, 1990 / L'Atelier imaginaire, 1991. L'Anonymat (páginas del Livre de l'hospitalité), pinturas de Francesca Chandon, París, 1991. Désir d'un commencement. Angoisse d'une seule fin, aguafuertes de Claude Garache, Saint-Clémentde-Rivière, Fata Morgana, 1991. Petites poésies pour les jours de pluie et de soleil, ilustraciones de Caroline Richard, París, Gallimard-Jeunesse, Folio Cadet Or, 1991.

${ }^{7}$ Texto de Edmond Jabès. Soprano, piano, violín, viola, violonchelo, flauta, clarinete, trompeta, trombón, trompa (18'). Interpretado por el Ensemble 2E2M, dirigido por Paul Méfano, en la Chapelle de la Sorbonne, París.

${ }^{8}$ París, Théâtre National de Chaillot. Obra para contralto, bajo, voz recitadora, flauta, tuba, trompa y live electronics. Basada en el Petit Livre de la subversion hors de soupçon. 16' 19'.

Por otra parte, en cuanto a otras obras inspiradas por textos de Jabès puede consultarse la introducción de Jean Frémon a Jabès (1992: 12-13), colección de sus escritos sobre artistas. En general por lo que respecta a la biografía y el progreso de la obra de Jabès puede consultarse Cahen (1991: 305-341) y del mismo autor "Écrire sa vie" en Jaron (1999: 17-68).

${ }^{9}$ Chillida realiza también en 1986 un dibujo a tinta de una mano como homenaje a Jabès (cfr. la reproducción en Maderuelo [2003b: 59]) y bajo ésta escribe en mayúsculas unas frases contando que 
rue de Téhéran ya bajo el nombre de Lelong ${ }^{10}$. La historia de este libro de Jabès comienza más de diez años antes con la publicación del poema Des deux mains, con papeles teñidos y dibujos de Raquel ${ }^{11}$, Malakoff, Orange Export Ltd. ${ }^{12}$ (Jabès, 1976), del que se hicieron 100 ejemplares en papel Arches firmados por el poeta y la pintora. Aunque, para ser exactos, el verdadero inicio habría que situarlo en 1975, ya que una parte del poema había aparecido en ese momento con una tirada de nueve ejemplares ${ }^{13}$. El texto de la edición de $1976^{14}$ constituirá el primer poema, "Ceux à qui...", del libro publicado por Lelong en 1986 ilustrado por Chillida ${ }^{15}$.

un día, tras una conversación con el poeta, le regaló un dibujo de una mano y que esto constituyó el punto de partida para la colaboración en La Mémoire et la Main.

${ }^{10}$ Daniel Lelong trabaja junto a Aimé Maeght y en 1964 se convierte en el director de la galería. En 1981, a la muerte de Aimé Maeght, la dirigirá junto a Jean Frémon y Jacques Dupin y se llamará Maeght-Lelong hasta que de forma definitiva en 1987 pasa a llamarse exclusivamente Galerie Lelong. Adrien, hijo de Aimé, abrirá su propia galería (con el mismo nombre de la primera, Galerie Maeght) en 1956, en la rue du Bac, dirigida actualmente por Isabelle Maeght, su hija.

${ }^{11}$ Cfr. Raquel, "Itinéraire". Raquel (Raquel Levy) nace en Gibraltar, de padre español. Pasa a Marruecos, estudiando en la escuela de Bellas Artes de Casablanca. Se traslada a París donde continúa sus estudios en L'École des Métiers d'Art y en la academia de la Grande Chaumière. Exposiciones importantes en la galería Arnaud (París, 1976), Garibaldi (Aix, 1981), Breteau (París, 1984). Exposición retrospectiva en Caracas en 1990. Crea junto a Emmanuel Hocquard la editorial Orange Export Ltd. Realiza la ilustración de numerosos libros a partir de 1969. Crea la revista Notes en 1986.

${ }^{12}$ Orange Export Ltd. (1969-1986) es, como decimos, una editorial creada por el poeta Emmanuel Hocquard y la artista plástica Raquel. Aun admitiendo diversas tendencias en su seno, fue punto de confluencia de ciertos intereses literarios reuniendo a autores como Anne-Marie Albiach, Jean Daive, Roger Giroux, Joseph Guglielmi, Emmanuel Hocquard, Pascal Quignard, Claude Royet-Journoud o Alain Veinstein. Cfr. Hocquard, E., Raquel et al. (1986).

${ }^{13}$ Puede leerse una descripción de esta publicación de Orange Export Ltd. en R. E. Stoddard, "Comment je lis Edmond Jabès: la réponse du bibliographe" (Lançon, 2007: 304-305). Pertenecía a la serie Chutes, libros muy breves elaborados manualmente por Hocquard y Raquel, tirados a nueve ejemplares. En esta edición no había ilustraciones.

${ }^{14}$ He podido consultar un ejemplar de la edición de 1976 (sin numerar ni firmar) posesión de Emmanuel Hocquard, quien tuvo la amabilidad de escanearlo y mandármelo, dada la gran dificultad para encontrar el libro en las bibliotecas de París. Por lo demás, son escasísimas las referencias al contenido real de esta publicación. Excepción son los estudios bibliográficos de R. E. Stoddard. En Stoddard (1998: 67 y ss), cuya entrada $30^{\mathrm{a}}$ trata de la edición que comentamos de 1976, precisa el autor que en la sección 4 se usa la forma "entrouvre" que luego cambiará a "entr'ouvre" y que los versos 2-6 de la sección 8 no serán retomados en la edición posterior, observación no exacta (que repite en Lançon [2007: 306]) ya que la mayor parte de lo que desaparece de esta sección pasará a un poema de la segunda parte de la edición de 1986 (vide infra). En la entrada 30b se estudia el libro en colaboración con Chillida de 1986 y aquí se lee: "previously published as Des deux mains, this edition and all subsequent ones published with the title La Mémoire et la Main add pt. II, 'Le sang ne lave pas le sang'. [...] Collected in Le seuil Le sable, 1990”. En realidad, no sólo se añade ahora la segunda sección de tal título sino también tres poemas que se suman a la primera sección, titulada, en efecto, "Des deux mains" y que son: "Main démasquée", “À Demi ouverte, ma main" y "Toujours cette image", como el mismo Stoddard aclara en Lançon (2007: 306), donde también se dedican unos párrafos a la edición de 1976.

${ }^{15}$ Las diferencias entre el texto del poema "Des deux mains" de 1976 y el de la edición posterior, se sitúan, como decíamos, en la octava sección, que presenta, en la primera edición, algunas líneas que 
Hay que señalar que la disposición (mise en page) del poema se mantiene prácticamente inalterable desde la primera entrega ${ }^{16}$. Por otra parte, las ilustraciones de Raquel en la edición de 1976 se distribuyen por grupos a página completa ${ }^{17}$ con un resultado de enorme fuerza y elegancia. Los papeles teñidos en tonos verdes y negros ofrecen sugerencias de dramatismo y profundidad que se alternan con líneas a lápiz, simples trazos aislados que recorren la página de arriba abajo (una sola vez aparece uno horizontal) evocando una despojada espiritualidad. Insinuaciones todas ellas en perfecta consonancia con el texto de Jabès.

El volumen con las ilustraciones de Chillida conocerá, un año después, en 1987, una nueva edición con reproducción no original de los aguafuertes en la editorial Fata Morgana: 970 ejemplares en papel verjurado marfil (30 ejemplares en Johannot fueron anunciados pero no realizados, según informa la editorial ${ }^{18}$ ). Algo más tarde, en 1990, estos textos aparecen, abandonando el complemento gráfico, en la recopilación de la poesía completa del autor para la colección Poésie/Gallimard, Le Seuil Le Sable. Poésies complètes 1943-1988 (Jabès, 1990). Hay que indicar que los poemas no sufren ninguna modificación desde la edición de 1986.

Observaremos ahora la funcionalidad estética de los elementos formales de ambas obras, partiendo del análisis de la primera edición, de 1986.

El libro, terminado en octubre de 1986, fue impreso tipográficamente en el taller de Robert Blanchet y en lo relativo a los grabados en el taller Hatz de San Sebastián. Blanchet, impresor y grabador en Boulogne-Billancourt, retoma el taller de Jacques Beltrand en 1952, realizando a lo largo de su carrera hermosos trabajos de bibliofilia. El taller Hatz (palabra que en euskera significa dedo y huella) fue fundado por Mónica Bergareche e Ignacio Chillida (tercer hijo del escultor, nacido en 1954) en 1976. En 1978, tras su boda, viajan a Barcelona y a París, donde trabajan con Fequet et Baudrier (especialistas en libros ilustrados) y con Robert Dutrou (grabador de artistas como Miró, Tàpies o Palazuelo), con quien colaboran de nuevo en Saint-Paul-de-Vence, volviendo luego a San Sebastián para relanzar

serán luego eliminadas tras el primer verso común ("main serrée sur sa faim”, único en la segunda edición): "ce poids, ce poing. // LE POINT. // ... Et qu'est-ce qu'un point sinon le trou vertigineux de toute fin? / - L'entrée visible?". Desde "Et qu'est-ce que..." el texto constituye el inicio del poema "Le trou", de la segunda parte del libro de 1986. Por otra parte, el libro en la edición de 1986 (y posteriores) se inicia con cuatro versos que no figuraban en 1976: "Il y eut, jadis, une main / pour nous conduire à la vie. // Un jour y aura-t-il une main / pour nous conduire à la mort?".

${ }^{16}$ Puede decirse que la mise en page de la edición artesanal de 1975, descrita en detalle por Stoddard, como decíamos, en Lançon (2007: 302), guía las posteriores con sus continuaciones.

${ }^{17}$ Tras la página 12 se sitúa una hoja con papel teñido, dos hojas de trazos a lápiz y otra hoja con papel teñido. Tras la 16 encontramos una hoja de trazos y una de papel teñido y tras la 20 , una hoja de papel teñido y una de trazo. La numeración no las considera.

${ }^{18}$ Cfr. Fata Morgana, "Livres. La mémoire et la main". 
Hatz, cuya andadura se extiende hasta el año 2000 y que realizará múltiples trabajos para Chillida y otros artistas ${ }^{19}$.

Como indica el colofón, se hicieron 125 libros (sin contar los $\mathrm{HC}$ y nominativos), con siete aguafuertes ${ }^{20}$.

En cuanto al soporte, se usó Muguet du moulin de Larroque, papel artesanal de un blanco muy hermoso, utilizado para la totalidad de los ejemplares. El libro está constituido por pliegos doblados por la mitad sin encuadernar. Estos se incluyen en una carpeta de papel con solapas en cuyo primer plano figura en gofrado hueco la estilización de una mano. El conjunto se guarda en una caja entelada color beige en cuyo primer plano figura otra mano estilizada (distinta a la anterior) también en gofrado hueco. En su lomo aparece, arriba, "JABÈS / CHILLIDA" en horizontal, luego, en disposición "francesa", de abajo a arriba, el título, también en mayúsculas, y finalmente, en el extremo inferior, en minúsculas y en horizontal, "lelong éditeur", todo con el mismo gofrado. El formato de las hojas es de 32 x $24^{\prime} 5 \mathrm{~cm}$.

En cuanto a los distintos elementos que entran en juego en la mise en page, comenzaré comentando la tipografía. Se han usado para todos los textos caracteres Imprint de cuerpo 14, letra de gran belleza, creada en 1912 por Monotype Corporation de Washington para la publicación periódica The Imprint de Londres (1913), consagrada a temas de tipografía e impresión en general. Es un tipo de la familia de las letras de transición (entre las garaldas y las modernas), inspirada en la dieciochesca Caslon, con modulación vertical. Carácter regular, proporcionado y de cierta robustez, se consideró desde el inicio como especialmente adecuado para su utilización sobre papeles antiguos y de arte. Percibimos, por otra parte, un generoso interletrado que otorga elegancia a la línea.

La disposición de los poemas en la página no es regular. Desplazándose de continuo respecto al centro, podemos ver los textos situados en los cuatro

\footnotetext{
${ }^{19}$ Cfr. el catálogo Departamento Cultural Caja de Ahorros Vizcaína (1982: 5). Entre los trabajos del taller Hatz, serán muy importantes los realizados para los libros ilustrados de Eduardo Chillida desde inicios de los años ochenta: Biblioteca Nacional (1982), Voz Acorde: Homenaje a Jorge Guillén; E. M. Cioran (1983), Ce maudit moi; E. M. Cioran (1985), Face aux instant; Edmond Jabès (1986), La Mémoire et la Main; Yves Bonnefoy (1986), Le miracle du feu; Yves Bonnefoy (1990), Une Hélène de vent ou de fumée; Eduardo Chillida y Francisco Calvo Serraller (1991), Zedatu; Joan Brossa (1994), A peu pel llibre; José Ángel Valente (1996), Cántigas de Alén; Eduardo Chillida et al. (1997), Hommage à Johann Sebastian Bach; Jorge Semprún (1997), L'écriture ou la vie; Clara Janés (1998), La indetenible quietud; Parménides (1999), Le poème de Parménide; Eduardo Chillida et al. (2000), Aromas.

${ }^{20}$ El ejemplar que he consultado es el $\mathrm{n}^{\mathrm{o}}$ 6, propiedad del Museo Chillida Leku, Hernani. Este museo desde 2010 está abierto sólo a investigadores. Aprovecho para expresar mi agradecimiento a Ignacio Chillida por su generosa amabilidad al haberme dedicado su tiempo en varias ocasiones. Todos los ejemplares van firmados por Chillida en el frontispicio y por los dos creadores en la página de colofón. Los cincuenta primeros van acompañados de una suite con los grabados, todos numerados y firmados por el autor. Además de esta tirada se hicieron veinte ejemplares fuera de comercio (HC) numerados de I a XX y algunos nominativos.
} 
cuadrantes de la página, predominando el comienzo en la parte superior. Ya indiqué que la mise en page de 1975-1976 es respetada en lo esencial, para el primer poema, en la edición del 86 y se continúa con esa misma dinámica para el resto. Además queda fijada, de forma que reaparece en la edición de 1987. Esta diversidad otorga movimiento a la compaginación e indudable belleza, lo que se alía a unos márgenes amplísimos para dar una respiración extraordinaria a cada página.

Pero donde se encuentra, en el plano formal, la mayor originalidad y el mayor rendimiento estético de esta obra es en la relación entre grabados y texto. Aquí prima igualmente la diversidad de soluciones. El primero aparece como frontispicio y en él figura, como parte integrante del aguafuerte, una parte del texto de Jabès escrita a mano, con letras mayúsculas y en espejo, por Chillida. El escrito proviene del final del poema de la primera parte "À demi ouverte, ma main" importante que encontramos en ésta y en las demás intervenciones de Chillida en el libro es la funcionalidad del borde de los grabados, la huella de la plancha, con formas originales, que marca en el papel un espacio y un relieve que serán utilizados estéticamente tanto en el anverso como en el reverso de la hoja. En el caso del frontispicio vemos que el rectángulo de la plancha termina por la derecha en dos círculos incompletos. El segundo grabado aparece en página par, solo, siendo dos estudios de dedos y una mano completa y presenta dos semicírculos hacia dentro por los lados. Sirve para enmarcar, con su relieve, el poema precedente, en página impar, "Il y eut jadis une main". Este aguafuerte en la edición de Fata Morgana pasa al final del libro. El tercer grabado se sitúa en la parte superior de la página, ocupando ésta, horizontalmente, de parte a parte. Esta vez hay dos semicírculos saliendo en la parte inferior. Más abajo aparece el texto: "On meurt de ses propres mains". El cuarto engloba la sección octava del primer poema, "VIII Main serrée sur sa faim" impreso dentro del rectángulo vertical del grabado con dos cuartos de círculo hacia dentro en sus esquinas inferiores. Tras la sección XIII, última del primer poema, llega el quinto grabado, solo en la página y que presenta la originalidad de que la parte inferior de la plancha está cortada como si fuera la base de la mano, como si ésta sobrepasara el marco para invadir la página. Al final del poema de la primera parte, "Toujours cette image", solo en la página y centrado, el sexto aguafuerte con semicírculos hacia dentro en los lados. Al final del poema de la segunda parte "Le trou" aparece el último grabado, solo en la base de la página, siendo cortada la plancha con un saliente centrado en la parte superior.

En la edición de 1987 se respeta la colocación de los grabados y del texto, salvo en el caso señalado. Por otra parte, no se han reproducido en ésta más que las líneas estrictas del dibujo de las manos de cada aguafuerte, es decir, no hay bordes de la

21 “('Qu'importe que ce soit de droite à / gauche ou de gauche à droite / la main n'écrit que dans le sens / brûlant de la vie à la mort, de l'aube / au crépuscule', disait-il. / 'Le jour et la nuit sont les deux / pôles d'une main', disait-il aussi." Indicaré también, en cada caso, el número de página del libro en la colección Poésie/Gallimard, edición aún hoy fácilmente accesible. Cfr. Jabès (1990: 374). 
plancha, con sus formas características, y tampoco se ha reproducido todo el entramado de vibraciones gráficas del fondo de los grabados, con decenas de tenues líneas y puntos aleatoriamente distribuidos y que dan vida a las obras, sobre todo en cuatro casos, en la edición anterior ${ }^{22}$. Esto no disminuye, ni mucho menos, la belleza de la edición de Fata Morgana. En su cubierta, en rojo sangre, se ha reproducido la mano de la carpeta interior de 1986. Sobre un papel verjurado color crema, en el interior, las manos de Chillida parecen flotar por la falta de marco, siendo el resultado global de gran fuerza estética.

En ambos casos, la unión de creación poética, plástica y tipográfica es fundamental, determinante. Evidentemente, si acudimos al librito de bolsillo de Gallimard, la fuerza de la palabra de Jabès no puede verse disminuida pero lo importante es lo que se añade en las otras ediciones, no lo que puede o no perderse. Lo que se suma es de enorme valor, la realidad de una comunicación profunda del núcleo de lo humano entre dos creadores y entre éstos y el espectador.

En $1985^{23}$ Jabès escribe un texto sobre Chillida para el catálogo de la exposición Europalia de Bruselas en el que hace referencia al cartel de Maeght y al libro para Lelong que estaban ya preparando, donde dice: "Frontières du visible et de l'invisible // C'est à ces limites que je retrouve Chillida!" (Jabès, 1992: 64). Lo visible, la materia, el cuerpo, la mano. Lo invisible, el espacio, el aire, el espíritu. La indagación, en efecto, sobre estas fronteras ha sido clave tanto en el desarrollo de la obra del poeta como en la del escultor.

Intentaré ahora esbozar algunas reflexiones sobre la interacción entre las manos realizadas por Chillida y los textos del libro de Jabès ${ }^{24}$.

El catálogo de Maderuelo consagrado a las manos de Chillida (2003b) se inicia con dos dibujos de 1945 y el de Schmalenbach (1979) con dos obras de $1949^{25}$. El

\footnotetext{
${ }^{22}$ Dichas vibraciones provienen, según me informa su grabador, por una parte, de la reutilización casi sistemática de planchas anteriores (una buena cantidad fue traída por éste de Saint-Paul-de-Vence) y por otro, de la acción abrasiva sobre las planchas barnizadas que Ignacio y Eduardo Chillida realizaban de muy variadas maneras, arrastrándolas, pisándolas, dejándolas a la intemperie, etc., de forma que quedaran huellas que pudieran tomar una cierta cantidad de tinta.

En cuanto a los cortes de la plancha, sigue diciéndome Ignacio Chillida, se realizaban con una sierra, manual o eléctrica, teniendo que ser luego los bordes cuidadosamente limados.

23 "Petite réflexion en marge de l'œuvre d'Eduardo Chillida", en Fernández et al. (1985: 24-25) y en Jabès (1992: 63-68), donde el título aparece en plural, "Petites réflexions...".

${ }^{24}$ Cabe preguntarse si la disposición de los grabados a lo largo del libro es aleatoria u obedece a determinada necesidad temática, además de responder a una consonancia global. Aunque no sea posible una respuesta taxativa, en cualquier caso, el lector es "guiado" por un orden texto-gráfica concreto, de modo que su sensibilidad, inevitablemente, une lo inmediatamente contiguo. El lector, por otra parte, tiene siempre derecho a poner en juego su imaginación y, en el caso de los libros ilustrados, es, precisamente, uno de los sentidos de la colaboración entre creadores el de multiplicar los ecos.

${ }^{25}$ Se trata de dos durmientes: el brazo y la mano de uno de ellos recuerdan poderosamente la famosa escultura Ilarik (1951, primera obra abstracta en hierro).
} 
escultor declara en 1959 (Esteban, 1971: 11) haber dibujado hasta esa fecha muchas $\operatorname{manos}^{26}$. Ese año el autor, que durante un largo periodo había evitado el contacto con lo griego, se queda fascinado en el Louvre ante la mano derecha de la Victoria de Samotracia ${ }^{27}$, descubierta en 1950 por Charbonneaux y expuesta a partir de 1954. Esto lo lleva a reconciliarse con la luz y las formas griegas sin sentirse amenazado por una posible influencia negativa de éstas y a viajar a Grecia con Dupin y Clayeux en 1963. Los dibujos de manos continúan en los sesenta y desde inicios de los setenta empiezan a aparecer las manos grabadas ${ }^{28}$. Cabe preguntarse si son las suyas manos griegas. La claridad de su dibujo, la importancia del contorno, la ausencia de toda sombra, la sutilidad de los pliegues, hacen pensar en los dibujos de los vasos áticos de figuras rojas. Parece como si Chillida, que se sabe artífice, como él mismo expresa, de la "luz negra", vasca, atlántica y acepta este destino desde Ilarik (1951), hubiese decidido guardar una parte griega, clara, al dibujar las manos. Por otra parte, sus alabastros de los años sesenta son igualmente una reconciliación con el mundo griego, estudios de la relación entre materia y luz, blanca en Grecia, gris en el Cantábrico ${ }^{29}$.

Las manos de Chillida presentan una enorme variedad formal pero indudablemente una disposición prevalece en ellas: la de la mano entreabierta. El autor busca el hueco, la concavidad de la mano, el espacio imaginario detrás de unos dedos que se tocan, que se cierran parcialmente.

Como indica Schmalenbach, en las manos de Chillida (dibujadas, pero lo mismo vale para las grabadas) no se da una búsqueda de mensaje y aún menos de pathos:

En su tratamiento artístico, los valores psíquicos o expresivos de la mano, cualquiera que éstos sean, no tienen relevancia. Para Chillida, las manos son básicamente dos cosas: fenómenos de movimiento y fenómenos de espacio. Y sólo como tales atraen reiteradamente nuestra atención [...]. Ningún otro artista, a lo largo de la historia, ha abordado este tema con pareja intensidad [...], no existe un ejemplo igual en el que la mano constituya un tema per se [...]. Sus manos no expresan nada, no son signos de nada, sino, pura y simplemente, formas en el espacio [...]. El juego dinámico de [los] pliegues [de la mano] es lo que fascina a Chillida. [El autor] elige en ocasiones complicadas posturas de los dedos con objeto de acentuar su carácter de acontecer en el espacio [...]. Las manos aparecen en fragmentos, no sólo están separadas del cuerpo sino que también ellas se deshacen [...] se desprecia la unidad orgánica [...]. Como escultor, Chillida no se ha ocupado de la mano [...]. Su obra toda se funde en la naturaleza. Sus propias manos serían para nuestro artista

\footnotetext{
${ }^{26}$ Maderuelo (2003b: 14) afirma que los dibujos (sin contar los grabados) de manos de Chillida son, en total, más de trescientos.

${ }^{27}$ Dice Claude Esteban (1971: 13) a propósito de ésta: "Cette main, que Chillida a reconnue, est bien autre chose qu'une forme admirable [...]. Elle semble ignorer [...] l'antagonisme entre l'être-là disparate et la pensée, la matière nocturne et le signe. Mais Chillida le sait aussi: elle est un 'don du ciel'. Non le fruit d'un savoir, mais une Grâce -accordée à ceux-là seuls que Chillida appelle des hommes de rupture".

${ }^{28}$ En total Chillida hizo 56 grabados de manos entre 1971 y el año 2000, según puede verse en los cuatro volúmenes del catálogo completo de la obra gráfica (Koeler, 1996-2001).

${ }^{29}$ Respecto a la mano del Louvre y la relación con Grecia cfr. Chillida (2003: 35-38).
} 
el pedazo de naturaleza más próximo. De aquí extrae Chillida fuerzas y conocimientos que, en definitiva, también enriquecen sus esculturas (Schmalenbach, 1979: 3-5).

Aún siendo plausible la interpretación del crítico, es preciso indicar que, en cualquier caso, la mano está cargada de significados por sí misma y que el escultor es plenamente consciente de ello. Podría decirse que éste no fuerza su significación sino que deja hablar a la mano. Por otra parte, Octavio Paz se ha expresado con total justeza sobre el problema del espacio en nuestro autor: "las formas quieren colonizar el espacio pero el triunfo del espacio es la anulación de las formas. Si Chillida fuese budista, se inclinaría por la victoria del espacio; es escultor [grabador, dibujante, añadiríamos] y aspira a expresar esa dualidad, no a disolverla (Paz, 1980: 11)". El poeta afirma que en las obras de Chillida la forma intenta apresar lo inaprensible e indecible, "el espacio puro, sin propiedades y sin límites" (Paz, 1980: 16) y concluye que "sus esculturas [sus grabados] son la casa del espacio y están habitadas por un ser plural y uno. Chillida lo llama el 'espacio interior' pero podría llamarse también vacuidad o dios o espíritu o logos o proporción” (Paz, 1980: 18).

Javier Maderuelo otorga a las manos del artista una importancia capital, centro de su universo formal ${ }^{30}$ :

Casi todas las obras que Eduardo Chillida ha realizado como escultor, como grabador o dibujante [...] tienen como referente último la mano [...]. [Sus manos son] líneas puras y autónomas, como trazos pulsionales que limitan el espacio del papel [...]. Pero, ¿por qué dibujar entonces tan concretamente una mano [...]? Muy probablemente, la primera experiencia de espacio que tiene un recién nacido se produce al extender la mano y sentir su profundidad y al intentar atraparlo cerrando los dedos. Así, la mano aprehende el espacio real de manera activa, esa misma actividad pretende ser traspasada al papel por Chilllida [...]. El origen iconográfico de muchas de las obras [del autor], tanto dibujos y grabados como esculturas, pueden ser resultado, consciente o inconsciente de la estilización de su propia mano [...]. Hay que insistir en que la idea de espacio de Chillida se concentra en la experiencia, personal e íntima, que surge de la sorpresa del reconocimiento de su propia mano (Maderuelo, 2003a: 24-26).

Es posible que esta idea sobre el origen iconográfico de buena parte de la obra del autor sea arriesgada y difícil de aplicar en muchos casos, pero es innegable que la forma de la mano, de los dedos, los óvalos de las uñas, las líneas del contorno del puño, etc., pueden rastrearse en bastantes grabados puramente abstractos. Por otra parte, si nos es permitido desarrollar como juego la imagen de la mano del niño ${ }^{31}$,

\footnotetext{
${ }^{30} \mathrm{Cfr}$. también la introducción del autor al catálogo ya citado (Maderuelo, 2003b), del que el texto de la revista de la Fundación Juan March es un amplio resumen.

${ }^{31}$ No se puede no citar al menos las palabras finales de Henri Focillon en su célebre ensayo de 1934 Éloge de la main: "La main arrache le toucher à sa passivité réceptive, elle l'organise pour l'expérience et pour l'action. Elle apprend à l'homme à posséder l'étendue, le poids, la densité, le nombre. Créant un univers inédit, elle y laisse partout son empreinte. Elle se mesure avec la matière qu'elle métamorphose, avec la forme qu'elle transfigure. Éducatrice de l'homme, elle le multiplie dans l'espace et dans le temps" (Focillon, 1981: 128).
} 
diríamos que esas manos que a veces se cierran y se abren continuamente, en rotación, de los recién nacidos, sin asir realmente nada, como puro gesto de conocimiento, de medida o de deseo, o que si agarran sueltan al poco por falta de fijación, serían como las manos de Chillida que no empuñan y muestran su hueco, lejos del aferramiento utilitario de la mano adulta. José Ángel Valente (Calvo Serraller et al., 1996: 117), en una conversación con Chillida, subraya la importancia de la actitud de escucha del artista, de espera atenta o de disponibilidad para el encuentro y la pone relación con "la mano que busca" de Picasso dibujando en el documental Le Mystère Picasso de Clouzot: "el pensamiento del pintor está en la mano, esa mano que piensa [...], [que] iba tanteando y provocaba entonces el encuentro con la forma".

Analicemos ahora los grabados en libre contrapunto con los textos. En el primero se contraen ligeramente las primeras falanges de una mano izquierda dispuesta hacia abajo. Con la cita del poema dejada en espejo, es un posible homenaje a Leonardo, autor, además, de famosos estudios de manos. El papelespejo se hace espacio que invierte y convierte, aún más, en algo misterioso o mágico la escritura ${ }^{32}$. La mano hacia abajo recuerda igualmente en su postura, si queremos continuar buscando resonancias clásicas, a la diestra del David de Miguel Angel. La escritura "en el sentido ardiente de la vida a la muerte" (vide nota 21), vendría a ser, siguiendo este eco, una fuerza que se opone a la oscuridad del gigante.

En el segundo grabado (que en 1987 pasa al final del libro) hay una mayor tensión formal, ya que junto a una mano derecha que se cierra (sobre todo el pulgar se ha cernido sobre la palma) aparecen dos fragmentos de mano: dos dedos a su izquierda y tres a su derecha ${ }^{33}$. Esta profusión de dedos con un cierto desorden en su colocación, crean una meditada extrañeza. Funciona como un segundo frontispicio, en el reverso del texto que Jabès sitúa en 1986 como introito al libro y que es enmarcado por la huella de la plancha. La mano completa del centro del aguafuerte aferra suavemente el vacío, es quizás la mano que nos conducirá a la muerte nombrada en los versos.

En el tercero, los cinco dedos de una mano derecha intentan reunirse suavemente en un punto, vuelta hacia arriba. Con el extremo de estos dedos se sostiene un ápice de algo que no vemos. El dibujo no es completamente realista ya que el pulgar busca el meñique de forma que éste queda en una torsión imposible. El resultado óptico es, sin embargo, el descrito. Recordemos de paso que Chillida a menudo dibujaba con la mano izquierda, siendo diestro, para evitar una excesiva perfección y exactitud y también que tomaba sus propias manos como modelo. Aquí nada atrapan los dedos sino el vacío. Lo que en Jabès es fuego, ceniza, sombra y

\footnotetext{
${ }^{32}$ No es la primera vez que Chillida usa textos en espejo. Varios grabados de manos los incorporan con anterioridad.

${ }^{33}$ No es una bi-esku, una doble mano, como otras realizadas por el autor.
} 
anulación, en Chillida es sólo cinco dedos que serenamente unen sus puntas. Y esta relación irradia.

El cuarto aguafuerte presenta una mano izquierda semicerrada y un tanto contorsionada por un leve giro hacia arriba, elemento de tensión que se alía al carácter siempre incompleto del dibujo. Chillida raramente dibuja una mano entera, lo hace de forma, podríamos decir, fragmentaria, como indicaba Schmalenbach (vide supra). La mano en sí es ya un fragmento, una parte esencial y misteriosa del hombre al que queda evocando (recordemos la potencia de la mano aislada del Louvre o la de las manos prehistóricas) y este carácter se intensifica más aún, como decimos, por su factura parcial e inconclusa. Volvemos a observar, por otra parte, la falta de realismo por la posición imposible de los dedos meñique y anular. Los dedos se reúnen intentando aferrar algo con las últimas falanges. Dentro del rectángulo del grabado se incluye la impresión de la octava parte del primer poema del libro (es decir, el texto no está integrado en la plancha, como en el frontispicio): "Main serrée sur sa faim". La expresión "rester sur sa faim", que significa quedar insatisfecho, se transforma aquí para mostrarnos en esta metáfora global una mano que busca y no encuentra.

Las secciones XI y XII del primer poema (núcleo originario del libro, no lo olvidemos) aportan una dimensión cósmica. El universo atraviesa la mano para luego caer, con ella, en el abismo. Una mano que parece sostenerlo todo sin en realidad sostener nada. El libro, la creación del hombre, la interpretación continua del mundo, se equiparan en elipsis con la noche estrellada, que Jabès sitúa en caída o en catástrofe. La sección XIII, en página par, se acompaña en la impar de la mano más hermosa del libro, cuya plancha, como dijimos, está cortada en su parte inferior figurando la base de aquélla y cuya huella en el papel la dibuja. El texto une la idea de luz a la de polvo, a la del origen de todo lo que existe, a la materia primera y la fusión atómica de las partículas. Luego la referencia de nuevo, en este memento mori, al término, al colapso gravitatorio de las estrellas y al final de la luz. La mano de Chillida, quinta de la serie, se alza, aparece en vertical, esboza quizás un gesto de autoprotección. Vuelve a ser una mano imposible: cada dedo parece tener una vida autónoma, girar con independencia de las leyes del movimiento del cuerpo. En cualquier caso, vuelve a aferrar levemente el espacio. Chillida se declara "enamorado del espacio" (Calvo Serraller et al., 1996: 103). El espacio es el aire, es la materia rápida (en palabras del escultor ${ }^{34}$ ) y también el vacío taoísta, el espíritu, el misterio.

\footnotetext{
${ }^{34}$ Por ejemplo en Chillida (2005: 54): "El diálogo limpio y neto que se produce entre la materia y el espacio, la maravilla de ese diálogo en el límite, creo que en una parte importante se debe a que quizás el espacio es una materia muy rápida, o bien la materia es un espacio muy lento".
} 
Aquí se cierra el primer poema. Éste, como hemos dicho, recibe cinco grabados de los siete del libro. El resto, la mayor parte de él, sólo incluye dos ${ }^{35}$. Los otros tres poemas de la primera parte sólo son acompañados por un grabado de cierre. El último de estos poemas, "Toujours cette image", lleno de un aire de esperanza, habla de unas manos que se unen y se elevan escapando al peso terreno. El grabado que sigue a estas palabras, sexto del conjunto, es el de una mano derecha en la que sólo se aprecia el pulgar. Los otros dedos, cerrados, se presentan exclusivamente por la línea de su contorno exterior como conjunto, creando de nuevo una fuerte sensación de extrañeza. "Rondes mains comblées". Mano que al fin se cierra sobre algo, aunque sea sobre "la page froissée" (Jabès, 1990: 376).

Es posible que Jabès tuviera en mente el poema de García Lorca, basado en el amor y el misterio, "Casida de la mano imposible", perteneciente al Diván del Tamarit ${ }^{36}$, cuando escribió su libro. Reproduzco algunos versos: "Yo no quiero más que una mano, / una mano herida, si es posible. / Yo no quiero más que una mano, / aunque pase mil noches sin lecho / [...] / sería el guardián que en la noche de mi tránsito / prohibiera en absoluto la entrada a la luna. / [...] / Yo no quiero más que esa mano / para tener un ala de mi muerte" (García Lorca, 1981: 86). El título del primer poema de la segunda parte de La Mémoire et la Main, "Main douce à la blessure même", podría ser una variación sobre Lorca como eco en la memoria.

En Jabès, el dolor y el misterio son los goznes sobre los que gira su universo de manos ${ }^{37}$. "Tant de larmes dans une main / pour abreuver la mort". "Une main, surgie du néant, / surplombe nos tombes". El vértigo de la existencia indigente, de la sed, de la muerte y del vacío se resumen en este nudo metafórico. Lanza su interrogación sobre la muerte desde la realidad del desierto como patria y Chillida, hombre religioso, responde con la sutilidad de una obra que se mueve, igualmente, en los límites y en la indagación, no en la afirmación.

El único grabado de la segunda parte se sitúa al final del poema "Le trou". Esta séptima es una mano derecha en la que la punta del pulgar busca el extremo del anular, sobresaliendo por en medio, hacia el centro de la palma, el dedo corazón. A la izquierda, el límite de la mano está figurado con una doble línea, lo que por un instante crea la ilusión de la presencia de dos manos. Chillida no dibuja nunca las

${ }^{35}$ Lo que justifica la decisión, en la edición de 1987, como decíamos, de llevar uno al final de la segunda parte.

${ }^{36}$ Libro publicado póstumo, en 1940, en Buenos Aires y Nueva York. Escrito probablemente entre 1931 y 1934 e inspirado en parte (a un nivel de superficie) por los Poemas arábigoandaluces publicados en 1930 por Emilio García Gómez. La poesía completa de Lorca traducida al francés estuvo disponible en Gallimard en la primera mitad de los años cincuenta.

${ }^{37}$ No es ahora nuestro propósito hacer un recorrido de las líneas temáticas del libro y establecer sus relaciones, aunque sí querría mencionar, por su importancia, las referencias a la crueldad humana, a la mano que sangra por la violencia. "Mains contre mains. / Toute la vie —ô ce sang ! — / s'égoutte d'une main ouverte" (Jabès, 1990: 380). El título de la segunda parte y última frase del libro es una proclama: "le sang ne lave pas le sang" (Jabès, 1990: 377 y 391). 
líneas de la palma ni las del centro de los dedos. El juego de líneas interiores no le interesa en absoluto. Un espacio limpio parece circular entre esas manos entreabiertas que tienden a un grado de transparencia. "Le vide, le vide toujours en deçà" (Jabes, 1990: 386) es el verso que precede el aguafuerte y el final del poema.

Me gustaría terminar con unas rápidas referencias a otras colaboraciones de Chillida con poetas de lengua francesa ${ }^{38}$ : André Frénaud (1966), Charles Racine (1975), Édith Boissonas (1975), Jacques Dupin (1986) e Yves Bonnefoy (1986 y $1990)^{39}$.

En el primer caso, primer libro encargado a Chillida por Maeght, se trata de una obra impresionante desde que se abre su caja y aparece una carpeta color crema con un gofrado en relieve positivo ocupando toda la parte superior. Los grabados (nueve aguafuertes) de una gran fuerza expresiva van siempre solos en la página con la particularidad, en dos ocasiones, de ocupar la doble página. Los poemas fueron compuestos en Caslon Elzevir, un hermoso carácter de transición, aunque quizás se usó un cuerpo, el 28, excesivamente grande para un formato de 36 × $30 \mathrm{~cm}$.

En 1975 aparece la colaboración con el poeta de origen suizo Charles Racine, de formato más pequeño de lo habitual para los libros de bibliofilia, $23,5 \times 18 \mathrm{~cm}$, con cuatro potentes grabados aislados en la página. También en este año, colabora con Édith Boissonas en la serie Paroles Peintes de O. Lazar-Vernet, reunión de artistas plásticos y escritores que desde 1962 a 1975 conoció cinco números. Éste es el quinto, con aguafuertes originales de Miró, Chillida, Bryen, Ubac, Bona, Matta, Lepatre, Tàpies y Wilfredo Lam ilustrando a Yves de Bayser, É. Boissonas, Guillevic, A. Liberati, Pieyre de Mandiargues, Bernard Noël, Ponge, D. Roche y J. Stefan. En hermoso papel vélin de Arches, con un formato de 38 × 28, fue tirado a 132 ejemplares.

En 1986 se publica Les mères, de Jacques Dupin, en Fata Morgana. En la información de la tirada incluida en el libro se nos dice que se habrían hecho 75 ejemplares réimposés (de mayor formato) con cinco grabados originales del escultor. El resto de la edición hasta mil ejemplares se hizo en un papel verjurado color marfil formato de $22 \times 14 \mathrm{~cm}$ con reproducción de los originales. Estos datos (también recogidos en diversos catálogos y bibliografías) contienen dos errores: las ilustraciones de la obra (edición normal) no son reproducción de grabados sino de dibujos y, además, no llegó a hacerse tirada de bibliofilia con grabados originales, según información directa del grabador y de la dirección de la editorial ${ }^{40}$. En

\footnotetext{
${ }^{38}$ En el catálogo Biblioteca Nacional (2007) se reflejan sólo las ediciones con obra original, de bibliofilia. La colaboración con la poeta suiza Boissonas no se incluye, evidentemente, por no tratarse de un libro completo sino de una parte. Tampoco aparece el libro de Dupin por no cumplir la condición inicial (vide infra).

${ }^{39}$ Cfr. sobre las analogías estéticas entre Bonnefoy y Chillida el artículo de Martínez (1995).

${ }^{40} \mathrm{Al}$ no poder encontrar ejemplares de esa tirada de tête y al no hallar en el catálogo razonado de la obra grabada ninguna mención de ellos, me puse en contacto con Ignacio Chillida, quien me informó de que no se habían realizado grabados sino que se enviaron dibujos y que no recordaba que
} 
cualquier caso, y hechas estas precisiones, quiero subrayar de nuevo la elegancia de los libros de Fata Morgana.

En este fecundo año, aparece también una extraordinaria colaboración con el poeta Yves Bonnefoy para François Bénichou. En primer lugar una elegante carpeta entelada dentro de una caja prepara la sorpresa de esta edición de enorme formato 65 x 50. Un serio Bodoni cuerpo 36 viste los poemas, acompañados de tres grabados. Hemos podido consultar el ejemplar número 10 con una dedicatoria del poeta al escultor: "Cher Eduardo Chillida, je suis heureux d'inscrire ici mon nom à côté du vôtre. Quelles belles gravures, qui ancrent et aèrent les mots! Avec beaucoup d'admiration et d'amitié, Yves B. Paris, le 5 novembre 1986". También para F. B., en 1990, vuelven a colaborar Bonnefoy y Chillida en el fastuoso libro en formato 66 x 52 Une Hélène de vent ou de fumée, con tres aguafuertes muy hermosos impresos por Hatz.

Entre todas estas obras asombrosas de colaboración con poetas de expresión francesa ${ }^{41}$, querría destacar, sin establecer comparación alguna, la fuerza de la sutil mise en page y la poética belleza objetual de La Mémoire et la Main en su edición de 1986.

\section{REFERENCIAS BIBLIOGRÁFICAS}

Biblioteca Nacional. Área de publicaciones, (2007) Los libros de artista de Chillida. Madrid, Biblioteca Nacional.

Boissonas, É. et al., (1975) Paroles Peintes V. París, O. Lazar-Vernet.

Bonnefoy, Y., (1986) Le miracle du feu. París, F. B.

Bonnefoy, Y., (1990) Une Hélène de vent ou de fumée. París, F. B.

Cahen, D., (1991) Edmond Jabès. París, Belfond.

Calvo Serraller, F., Chillida, E. \& J. A. Valente, (1996) "El arte como vacío" in Revista de Occidente. № 181, pp. 99-117.

Chillida, E. et al., (2003) Elogio del horizonte. Barcelona, Destino.

Chillida, E., (2005) Escritos. Madrid, La Fábrica.

Departamento Cultural Caja de Ahorros Vizcaína, (1982) Hatz. El taller calcográfico de Ignacio Chillida. Bilbao, Caja de Ahorros Vizcaína.

Dupin, J., (1986) Les mères. Saint-Clément-de-Rivière, Fata Morgana.

Esteban, Cl., (1971) Chillida. París, Maeght.

Férnandez, A., Jabès, E. et al., (1985) Chillida. Europalia '85 España. Bruselas, Musée d'Art Moderne.

Focillon, H., (1981) Vie des formes, suivi de Éloge de la main. París, PUF.

se hubiese hecho edición alguna de bibliofilia. David Massabuau, director de Fata Morgana, me comunicó que, a lo que parece, la tirada de lujo nunca existió.

${ }^{41}$ Consultadas en Hernani, en Chillida Leku, y en París, en la Bibliothèque Jacques Doucet. 
Fata Morgana, "Livres. La Mémoire et la Main" [En línea]. Disponible en: http://www.fatamorgana.fr/livres/la-memoire-et-la-main [Último acceso el 1 de mayo de 2013].

Frénaud, A., (1966) Le chemin des devins, suivi de Ménerbes. París, Maeght.

García Lorca, F., (1981) Diván del Tamarit. Llanto por I. Sánchez Mejías. Sonetos. Madrid, Alianza.

Hocquard, E., Raquel et al., (1986) Orange Export Ltd. (1969-1986). París, Flammarion.

Jabès, E., (1975) Des deux mains. Malakoff, Orange Export Ltd., col. Chutes.

Jabès, E., (1976) Des deux mains. Malakoff, Orange Export Ltd.

Jabès, E., (1986) La Mémoire et la Main. París, Lelong.

Jabès, E., (1987) La Mémoire et la Main. Saint-Clément-de-Rivière, Fata Morgana.

Jabès, E., (1990) Le Seuil Le Sable. París, Gallimard.

Jabès, E., (1992) Un regard. Saint-Clément-de-Rivière, Fata Morgana.

Jaron, S. (dir.), (1999) Portrait(s) d'Edmond Jabès. París, Bibliothèque Nationale de France.

Koelen, M. van der (ed.), (1996-2001) Eduardo Chillida. Opus I-IV. Catálogo completo de la obra gráfica. Mainz-Munich, Chorus Verlag.

Lançon, D. \& C. Mayaux, (2007) Edmond Jabès: l'éclosion des énigmes. SaintDenis, Presses Universitaires de Vincennes.

Maderuelo, J., (2003a) "De la mano al espacio" in Boletín Fundación Juan March. $\mathrm{N}^{\circ} 329$, pp. 24-26.

Maderuelo, J., (2003b) Chillida. Elogio de la mano. Madrid, Fundación Juan March.

Maeght Éditions, "Chillida. Placard Jabès" [En línea]. Disponible en: http://www.maeght.com/editions [Último acceso el 1 de mayo de 2013].

Martínez, P., (1995) "Yves Bonnefoy face à Eduardo Chillida: poétiques de l'objet sensible" in Revista de Filología Francesa. № 8, pp. 83-99.

Museo Chillida Leku, "Biografía" [En línea]. Disponible en: http://www.museochillidaleku.com [Último acceso el 1 de mayo de 2013].

Paz, O., (1980) Chillida. Barcelona, Ediciones 62.

Racine, Ch., (1975) Le sujet est la clairière de son corps. París, Maeght.

Raquel, "Itinéraire" [En línea]. Disponible en: http://www.raquellevy.com [Último acceso 1 de mayo 2013].

Schmalenbach, W., (1979) Eduardo Chillida. Dibujos: manos 1949-74. Barcelona, Polígrafa.

Stoddard, R. E., (1998) Edmond Jabès in Bibliography. A Preliminary Record of the Printed Books. París-Caen, Lettres Modernes Minard, col. Les carnets bibliographiques de la revue des lettres modernes. 